\title{
Does BCG vaccine prevent tuberculous meningitis?
}

\author{
N Thilothammal, P V Krishnamurthy, Desmond K Runyan, K Banu
}

\begin{abstract}
The reported efficacy of BCG vaccine in preventing pulmonary tuberculosis varies from $0-80 \%$; however, its efficacy in preventing tuberculous meningitis ranges from $52 \%-84 \%$. A case-control study was conducted to assess the efficacy of BCG in preventing tuberculous meningitis in children. New cases of tuberculous meningitis, confirmed bacteriologically, were registered as cases. Controls were children suffering from febrile convulsions attending the same hospital. A total of 107 cases and 321 controls, block matched for age, were registered. Vaccination status was determined from the history reported by the mother and by BCG scar reading. Data regarding socioeconomic status, crowding, and nutritional status were collected. Using multiple logistic regression analysis the odds ratio obtained for the presence of BCG scar was 0.23 (95\% confidence interval (CI) 0.14 to $0 \cdot 37$ ) and the protective efficacy of BCG vaccine in preventing tuberculous meningitis in children was found to be $77 \%(95 \%$ CI 71 to $83 \%)$. (Arch Dis Child 1996; 74: 144-147)
\end{abstract}

Keywords: tuberculous meningitis, BCG, vaccine efficacy, case-control study.

Tuberculosis continues to be a widespread disease especially in developing countries. Tuberculous meningitis, a serious complication of this infection, occurs in $7-12 \%$ of patients with tuberculosis and it is more common in children. ${ }^{1}$ In children, even with treatment, the mortality of tuberculous meningitis is $27 \%$ and permanent disability is estimated to be as high as $39 \%{ }^{2}$ BCG vaccination to control tuberculosis is still widely being used around the world, but conflicting results from several large trials have cast doubt about its efficacy in preventing pulmonary tuberculosis. ${ }^{3-6}$ The Tuberculosis Prevention Trial, conducted at Chenglepet District, Madras did not show any protective efficacy in preventing pulmonary tuberculosis. ${ }^{3}$ However, no special effort was made in this trial to ascertain the efficacy of BCG vaccine in the prevention of extrapulmonary tuberculosis in general or tuberculous meningitis in particular. The results from other studies undertaken to find out the efficacy of BCG in preventing tuberculous meningitis have been encouraging and the protective efficacy has ranged from $52-84 \%{ }^{7-10}$ It is possible that BCG can prevent tuberculous meningitis by preventing dissemination of the tubercle bacillus within the body even if entry is not prevented. ${ }^{11}$
A prospective study with sufficient power to establish efficacy is not easy to conduct. Hence a case-control study was designed to find out the efficacy of BCG vaccine in preventing tuberculous meningitis. This study also assessed the role of poor nutrition and low socioeconomic status in the development of tuberculous meningitis.

\section{Patients and methods}

SELECTION OF CASES

This study was conducted at the Institute of Child Health and Hospital for Children, Madras, from August 1990-December 1992. Children aged 12 years or less were included in the study.

Cases were selected from the inpatient department of the hospital. Inclusion criteria consisted of (a) two or more of the following clinical features, with or without fever: altered sensorium varying from drowsiness to coma, headache, vomiting, history of convulsions, meningeal signs, bulging fontanelle, papilloedema, cranial nerve palsy, and motor weakness of the limbs, and (b) bacteriological confirmation in the cerebrospinal fluid of acid fast bacilli. Children with the following conditions were excluded: tuberculous meningitis treated before referral, chronic medical illness or malignancy, and current immunosuppressive treatment.

During the study period, 406 cases clinically suspected to be tuberculous meningitis were admitted to the hospital. These cases were examined in detail after getting informed parental consent. Particulars regarding demographic data and BCG vaccination during the neonatal period were elicited from the mothers by a single medical officer throughout the study period.

Bacteriological examination of the cerebrospinal fluid was carried out at the Tuberculosis Research Centre, Madras for all these cases. Acid fact bacilli were demonstrated, by culture and/or smear, in the cerebrospinal fluid of 107 subjects who were registered as cases. These children were also screened with chest radiography for evidence of pulmonary tuberculosis.

\section{SELECTION OF CONTROLS}

Children attending the seizure clinic at the same hospital during the same period and who had febrile convulsions without other neurological abnormality were eligible control subjects. After applying suitable exclusion criteria as for cases, 321 controls were selected. Cases and controls were block matched for age and sex. Age matching was done within a range 
of \pm 2 months for children less than 2 years and \pm 6 months in older children.

Cerebrospinal fluid analysis was not done routinely for all controls. The ratio between cases and controls was 1:3. The power of the study was $80 \%$ to detect an odds ratio (OR) of $0 \cdot 5$. A total of 321 controls were registered.

\section{MEASUREMENT}

A precoded questionnaire was used to collect data from cases and controls. The main outcome measure, exposure to BCG vaccination, was assessed from (a) BCG scar, (b) history of vaccination as reported by the mother, and (c) medical records if available. The presence of a BCG scar was determined independently by a medical officer and also by a trained paramedical worker, at the time of first contact with the patient. Exposure to BCG vaccination was classified as definite and probable. Definite exposure indicated that both a BCG scar and positive maternal history of BCG vaccination were present. Probable exposure indicated that either a BCG scar or maternal history of BCG vaccination was present. The scar reading made by the paramedical worker was used to classify the vaccination status.

Nutritional status of the children was classified as recommended by a subcommittee of the Indian Academy of Pediatrics. ${ }^{12}$ According to this, children were normal when weight was $>80 \%$ of expected weight for their height, mildly malnourished when weight was $70-80 \%$ of expected weight for their height, moderately malnourished when weight was $60-70 \%$ of expected weight for their height, and severely malnourished when weight was $<60 \%$ of expected weight for their height.

Crowding was assessed by showing the parent of the subjects rooms of known area and then asking them to specify which room was nearest in size to their own house. The total number of people living in the house was noted and living space, as square foot per head, was calculated.

Type of house was defined as kutcha or pucca. A pucca house was one that had brick walls, cement flooring, and roofing other than thatched. When any one of the above was absent the house was said to be kutcha. This proxy measure was used to assess socioeconomic status.

The mothers were asked about their educational status and were classified as follows: never attended school, attended elementary school, attended high school or college.

\section{STATISTICAL METHODS}

Univariate analysis and multiple logistic regression analysis were carried out. Presence of a BCG scar was taken as an independent variable and disease status (tuberculous meningitis) as the dependent variable in this model. Covariates of interest in the regression analysis included mothers' education, type of house, nutritional status, and living space per head. BMDP statistical software was used.

\section{Results}

The age of the 107 patients ranged from 6 months to 12 years. The number of children under 5 years was $74(69 \%)$, and boys constituted $63(59 \%)$ of the total. The mothers of 75 $(70 \%)$ of our cases never attended school. A large proportion of patients $(78,73 \%)$ were living in kutcha houses. The vast majority $(94 \%)$ of cases and controls were living in houses with an area of less than 100 square feet. Cases and controls were comparable with respect to different characteristics (table 1).

Among cases, the chest radiograph was normal in $71(66 \%)$, pneumonitis was present in $18(17 \%)$, bronchopneumonia was present in five $(5 \%)$, and miliary tuberculosis in five (5\%). Hilar adenitis or thickened pleura were seen in eight $(7 \%)$ cases. Mantoux skin test was positive $(>10 \mathrm{~mm})$ in $13 \%$ of cases. Acid fast bacilli were demonstrated in cerebrospinal fluid by culture alone in $93(87 \%)$ cases, by smear alone in eight ( $7 \%$ ) cases, and by both smear and culture in six $(6 \%)$ cases.

The chance corrected agreement ( $\kappa$ ) for scar reading between the medical officer and the paramedical worker was $0 \cdot 8$. A BCG scar was present in 35 (33\%) out of 107 cases and 219 $(68 \%)$ out of 321 controls. From the mother's history, exposure to BCG vaccination was positive in $53(50 \%)$ cases out of 107 , and 259 $(81 \%)$ controls out of 321 . In 81 cases and 265 controls scar status and maternal history for BCG vaccination were in total agreement. Among this group, 31 (38\%) out of 81 cases and $211(80 \%)$ out of 265 controls had both a BCG scar and a positive maternal history for $B C G$ vaccination.

The effect of BCG vaccine upon risk of development of tuberculous meningitis was estimated separately by univariate analysis and through multiple logistic regression analysis. When the BCG scar was taken as the measure of vaccination status, the OR was $0.23(95 \%$ confidence interval (CI) 0.14 to 0.37 ) and the

Table 1 Characteristics of cases and controls; values are number (\%)

\begin{tabular}{lcc}
\hline Characteristic & $\begin{array}{l}\text { Cases } \\
(n=107)\end{array}$ & $\begin{array}{c}\text { Hospital } \\
\text { controls } \\
(n=321)\end{array}$ \\
\hline Age (years) & $36(33)$ & $106(33)$ \\
$<2$ & $38(36)$ & $123(38)$ \\
$2-<5$ & $21(20)$ & $60(19)$ \\
$5-<8$ & $12(11)$ & $32(10)$ \\
8-12 & $63(59)$ & $193(60)$ \\
Sex & $44(41)$ & $128(40)$ \\
Male & & \\
Female & $75(70)$ & $88(28)$ \\
Socioeconomic status & $18(17)$ & $69(22)$ \\
(1) Mother's education & $14(13)$ & $164(50)$ \\
Never attended school & $78(73)$ & $143(45)$ \\
Attended primary school & $29(27)$ & $178(55)$ \\
Attended high school/college & & \\
(2) Type of house & & \\
Kutcha & & \\
Pucca & $71(66)$ & $187(58)$ \\
(3) Crowding: living area (sq ft) & $6(28)$ & $114(36)$ \\
$\quad<50$ & $6(6)$ & $20(6)$ \\
$\quad 50-100$ & $12(11)$ & $5(2)$ \\
>100 & $22(21)$ & $11(3)$ \\
(4) Nutritional status & $64(41)$ & $64(20)$ \\
$<60$ & $29(27)$ & $241(75)$ \\
$\quad 61-70$ & & \\
$71-80$ & &
\end{tabular}

^Actual weight expressed as a percentage of expected weight for their height. 
Table 2 Efficacy of BCG in preventing tuberculous meningitis in various age groups

\begin{tabular}{lll}
\hline Age (years) & OR (95\% CI) & Efficacy (95\% CI) \\
\hline$<2$ & $0 \cdot 18(0 \cdot 08$ to $0 \cdot 44)$ & $82 \%(75$ to 90$)$ \\
$2-<5$ & $0 \cdot 21(0 \cdot 09$ to $0 \cdot 48)$ & $79 \%(70$ to 87$)$ \\
$5-<8$ & $0 \cdot 17(0 \cdot 05$ to $0 \cdot 58)$ & $83 \%(74$ to 94$)$ \\
$8-12$ & $0 \cdot 81(0 \cdot 17$ to $3 \cdot 73)$ & $19 \%(0$ to $>100)$
\end{tabular}

vaccine efficacy was $77 \%$ (95\% CI 71 to $83 \%)$. When exposure to BCG vaccination was assessed by maternal history alone, the OR was $0.23(95 \%$ CI 0.14 to 0.39$)$ and the vaccine efficacy was $77 \%$ (95\% CI 71 to $84 \%$ ). When both the BCG scar and a positive maternal history of BCG vaccination were taken together as evidence of exposure, the OR was $0.16(95 \%$ CI $0 \cdot 10$ to $0 \cdot 27)$ and the vaccine efficacy was $84 \%$ (95\% CI 80 to $88 \%)$. The OR for BCG vaccination was calculated for various age groups (table 2 ).

Among cases, 34 (32\%) were moderately to severely malnourished, whereas among controls only $16(5 \%)$ were moderately to severely malnourished. The OR according to nutritional status was calculated. The $\mathrm{OR}$ for severe and moderate malnutrition was 0.37 (95\% CI 0.09 to 1.48$)$, for mild malnutrition $0.17(95 \% \mathrm{CI}$ 0.07 to 0.43 ), and for normal nutritional status $0.22(95 \%$ CI 0.09 to 0.54$)$. The protective efficacy of BCG was $63 \%$ (95\% CI 31 to 127$)$ in moderately to severely malnourished patients, $83 \%$ (95\% CI 76 to 91) in mildly malnourished, and $78 \%$ (95\% CI 68 to 89$)$ in patients with normal nutritional status.

Using logistic regression analysis, the $O R$ obtained for presence of BCG scar was 0.28 (95\% CI 0.16 to 0.47 ), for better nutritional status $0.16(95 \%$ CI 0.05 to 0.59$)$, for literate mothers 0.36 ( $95 \%$ CI 0.26 to 0.51 ), for better house type 0.52 (95\% CI 0.29 to 0.93 ), and for more living space per head 1.64 (95\% CI 1.02 to $2 \cdot 64$ ).

The OR for the BCG scar estimated from multiple regression analysis was almost the same as the OR estimated from univariate analysis. Various regression models were tried and the OR for presence of BCG scar was not affected much by the presence of other variables in the model, for example nutritional status, type of house, and mother's education.

\section{Discussion}

The efficacy of BCG vaccine in preventing tuberculous meningitis was $77 \%$ in this study. This observation is consistent with that of others. ${ }^{8-10}$ Myint et al, however, reported that BCG vaccine efficacy in preventing tuberculous meningitis in children was $52 \%{ }^{7}$ In the present study among children below 8 years the efficacy ranged from $79 \%$ to $82 \%$, but in children above 8 years the efficacy was only $19 \%$. This could have been due partially to misclassification of the BCG status as well as waning of BCG efficacy with time. ${ }^{13}$ However, the number of children in this group was too small to draw any definite conclusion. The protective efficacy of BCG was found to be low in the moderately and severely malnourished group. The influence of nutrition on the efficacy of BCG vaccine is poorly understood and needs to be evaluated further. Literacy of mothers appeared to be an important risk factor and this has to be assessed further in future studies. The OR for better house type was 0.52 but the OR for more living space per head was $1 \cdot 64$. These contradicting values could have been due to misclassification error as a result of the wrong estimation of living space. In the present study, type of house is a reliable parameter compared with living space per head as the latter involved the parent's judgment about space. The OR for BCG vaccination was not much different in the presence of other parameters for different regression models.

In case-control studies, case definition is very crucial. Earlier studies defined cases either clinically or used a combination of clinical and bacteriological criteria other than cerebrospinal fluid culture. However, this study used a more specific case diagnosis, demonstration of acid fast bacilli in cerebrospinal fluid, as the case definition of tuberculous meningitis, thus decreasing misclassification bias.

Another potential source of bias is the ascertainment of vaccination status. History of vaccination, without evidence of a scar, must be interpreted cautiously. Additionally, a BCG scar may disappear over time. In order to minimise this error, vaccination status was classified into two categories: definite exposure and probable exposure. The protective efficacy was almost similar in these two groups, $84 \%$ and $77 \%$ respectively, indicating that such a misclassification was less likely to have occurred in this study. Two more precautions were taken to minimise measurement error while identifying BCG scar. The paramedical worker who examined the children for BCG scar was unaware of the null hypothesis and the scar reading was carried out before cerebrospinal fluid culture results were available.

Ideal control subjects would have been neighbourhood controls. But, with the available limited resources, identifying and recruiting matched neighbourhood controls for cases coming from different parts of the state was a limiting factor. Hospital controls were more likely to have received BCG vaccine than neighbourhood controls. However, in this study the proportion of children who contacted the doctor at least once for other vaccinations like diphtheria, tetanus, and pertussis and oral polio virus were equal in cases and controls. Thus it appears that cases were comparable with controls with respect to opportunities for being vaccinated with BCG. Cases and controls were also similar with reference to age, sex, and crowding.

This study shows BCG vaccine to be $77 \%$ efficacious in preventing tuberculous meningitis in children. Regardless of the protective efficacy of BCG in preventing pulmonary tuberculosis, in areas in which tuberculosis affects a significant percentage of the population, continued use of BCG may be warranted to prevent tuberculous meningitis.

We acknowledge the International Clinical Epidemiology Network (INCLEN) and Pharmacoepidemiology Group, 
We thank the Tuberculosis Research Centre - Indian Council of Medical Research, Madras for their valuable help in carrying out bacteriological investigations on cerebrospinal fluid samples. College and $\mathrm{Dr} \mathrm{K}$ G Kamal Child Health and Hospital for Children, for their valuable guidance.

1 Tandon PN. Tuberculous meningitis. In: Vinken PJ, Bruyn GW, eds. Handbook of clinical neurology. Vol 33. GW, eds. Handbook of clinical neurology: Vol 33. 2 Padma R, Duraipandian M, Nagarajan M, Prabhakar R, Ramakrishnan CV, Tripathy SP. Three chemotherapy studies of tuberc.

3 Tuberculosis prevention trial, Madras. Indian $\mathrm{f}$ Med Res 1980; 72 (suppl): $1-74$

4 Ten Dam H, Toman K, Hitze K, et al. Present knowledge of immunization against tuberculosis. Bull World Health Organ 1976; 54: 255-69.

5 Eickhoff T. The current status of BCG immunization against tuberculosis. Annu Rev Med 1977; 28: 411-23.
6 Clemens JD, Chuong JJH, Feinstein AR. The BCG controversy - a methodological and statistical reappraisal. $¥ A M A$ 1983; 289: 2362-9.

7 Myint TT, Win H, Aye HH, Kyaw-Mint TO. Case-control study on evaluation of BCG vaccination of newborn in Rangoon, Burma. Ann Trop Paediatr 1987; 7: 159-66.

8 Filho VW, Castilho EA, Rodrigues LC, Huttly SRA. Effectiveness of BCG vaccination against tuberculous meningitis: a case-control study in Sao Paulo, Brazil. Bull World Health Organ 1990; 68: 69-74.

9 Sharma RS, Srivastava DK, Singh AA, et al. Epidemiological evaluation of BCG vaccine efficacy in Delhi, 1989.

10 Camargos PA, Guimaraes MDC, Antunes CMF. Risk assessment for acquiring meningitis tuberculosis among children not vaccinated with BCG: a case-control study. Int $\mathcal{F}$ Epidemiol 1988; 17: 193-7.

11 Report of a WHO study group. BCG vaccination policies. World Health Organ Tech Rep Ser 1980; 652: 5-17.

12 Ghai OP. Nutrition during infancy. In: Ghai OP, ed. Essential pediatrics. New Delhi: Interprint, 1990: 37-45.

13 Report of a scientific group. Vaccination against tuberculosis. World Health Organ Tech Rep Ser 1980; 651: 7-21. 